\title{
Lógica de Serviço Aplicada a Concepção Arquitetônica
}

\author{
Service Logic Applied in the Architectural Conception
}

\author{
NOGUEIRA, Pablo F. D.; Mestre; Centro Universitário de João Pessoa \\ pablo.nogueira@unipe.br \\ OLIVEIRA, Estela Maris de M.; Mestra; Centro Universitário de João Pessoa \\ estela.oliveira@unipe.br
}

\section{Resumo}

Esta publicação pretende apresentar, a partir de uma abordagem prática demonstrada através de um estudo de caso, uma visão geral da lógica de serviço aplicada ao projeto arquitetônico e como esses princípios podem suplantar características programáticas para uma nova perspectiva no desenvolvimento de projetos. O foco principal é apresentar o início de um trabalho que está sendo realizado em ambiente acadêmico por professores e graduandos do Curso Superior de Tecnologia em Design de Interiores do Centro Universitário de João Pessoa, Paraíba, que utiliza como referência uma experiência de sucesso desenvolvido pela Aalto University, na cidade de Espoo, na Finlândia. 0 objetivo desta produção é expandir a discussão sobre métodos de design para projeto de ambientes a partir da abordagem de lógica de serviço.

Palavras Chave: design de serviço; lógica de serviço; design de interiores.

\begin{abstract}
This publication aims to present, from a practical approach demonstrated through a case study, an overview of the service logic applied to the architectural design, and how these principles can supplant programmatic characteristics for a new perspective in the development of projects. The main focus is to present the start of a work that is being carried out in an academic environment by professors and undergraduates of the Higher Course of Technology in Interior Design of the Centro Universitário de João Pessoa, Paraíba, which uses as reference a successful experience developed by Aalto University in Espoo, Finland. The purpose of this production is to expand the discussion about design methods for designing environments from the service logic approach.
\end{abstract}

Keywords: service design; service logic; interior design.

\section{A Lógica de Serviço e sua Aplicação na Arquitetura}

\subsection{A Lógica Dominante}

Nos últimos anos temos observado grandes mudanças socioeconômicas em nível global. A lógica dominante dos bens vem sendo substituída pela lógica dominante do serviço. A lógica dos bens baseada no desenvolvimento, produção e troca de produtos tangíveis vem perdendo espaço para a lógica em que as organizações, mercados e sociedade estão dispostos e atentos a troca de conhecimentos e habilidades (VLADYKINA, 2016, p.11). Ainda segundo Vladykina (2016), na lógica de bens o consumidor era apenas um usuário passivo de produtos criados pelas companhias, 
enquanto na lógica de serviço o consumidor passa a ser um stakeholder ativo com habilidade de ensinar as companhias o modo como atender as suas necessidades ao mesmo tempo que estes usuários não entenderão ou saberão exatamente o que querem (VLADYKINA, 2016, p.12). Desse modo podemos assimilar a citação de Lush e Vargo quando afirmam que muito em breve "o que é conhecido será menos importante porque o conhecimento estará atrasado e não será apropriado" (VLADYKINA, 2016, p.12 apud. LUSH e VARGO, 2014) entendendo que o aprendizado possuirá uma relevância maior do que o conhecimento. Nesse sentido, Vladykina (2016) considera ainda que mesmo que as experiências não sejam tangíveis, elas são mais valorizadas pelas pessoas do que os bens. Pois as pessoas consideram que o valor das emoções e habilidade em resgatar memórias superam o da qualidade de produção (VLADYKINA, 2016, p.13). Nesse sentido, pode-se entender que a lógica de serviço é capaz de reconhecer que o valor dos benefícios obtidos por determinado bem é maior que a posse dele.

Embora o entendimento sobre os ecossistemas baseados na lógica serviço tenha aumentado, também tem aumentado a oportunidade de aprofundamento dos estudos no entendimento sobra a criação de valor. Segundo Lusch et al. (2016), esse entendimento é desafiador porque as possibilidades de aplicação não têm limite fixo e sua expansão é constante (LUSCH et al., 2016, p.2960).

\subsection{Design thinking de serviços}

Diante deste panorama de substituição de bens de consumo pela busca de valores intangíveis em suas relações, o design thinking se apresenta como um modelo de trabalho que valoriza os novos paradigmas da sociedade contemporânea. Caracterizado pelo pensamento abdutivo, o processo trabalha a busca por soluções a partir do conhecimento aprofundado no universo que envolve o problema, de modo holístico e subjetivo. A empatia é utilizada no momento de imersão a fim de favorecer a apreensão dos fenômenos que ocorrem na natureza da questão.

A ênfase na experiência do usuário é a marca maior do design thinking, visto que considera suas relações reais, visão de mundo e sentimentos, o universo de pesquisa para ideias eficientes e personalizadas. Somado a isto, o modelo valoriza o envolvimento dos diferentes atores tanto na imersão, quanto na fase de ideação de modo a estimular os insights criativos não apenas no designer. Este profissional é encarado como um gestor do processo de design que articula as ações de pesquisa, análise e ideação, a partir da colaboração de designers e usuários. O processo, além de colaborativo, é interativo e flexível, o que permite que novas perspectivas de projeto sejam constantemente consideradas (VIANNA, 2012).

A partir da metodologia do design thinking, o design de serviços surge como uma abordagem direcionada ao desenvolvimento de ambientes a partir de modelos de processos, técnicas e ferramentas mais eficientes, leais e personalizadas às necessidades dos usuários de espaços de varejos, transportes, saúde, ensino, entre outros. O foco é a criação de experiências por meio da articulação de mídias tangíveis e intangíveis tendo cinco princípios fundamentais: a cocriação entre os diferentes grupos de usuários; o design centrado na experiência do usuário; a sequencialidade da linha de tempo da interação; a continuidade da experiência do serviço para o pós-serviço; e o pensamento no panorama geral do serviço, considerando os valores da marca (STICKDORN, 2014).

\subsection{O Estudo de Caso na Aalto University e o School-as-a-Service}

As mudanças socioeconômicas em nível global têm também afetado significativamente o 
modo como ocupamos o espaço. No ano de 2014 foi realizado na Aalto University em colaboração com o Royal College of Arts de Londres a aplicação de um curso que propunha a análise da relação do aprendizado em ambientes escolares para a cidade de Espoo, Finlândia. Desse modo, foi ofertada uma disciplina no curso de mestrado em arquitetura em colaboração com a Cidade de Espoo que propunha buscar novas soluções para estudantes e professores daquela cidade enquanto a instituição estivesse sob reparo. Naquela época a falta de infraestrutura para abrigar mais de 3.000 jovens estudantes de ensino fundamental forçou a disponibilização containers como instalações temporárias para salas de aula (VLADYKINA, 2016).

Segundo Jarmo Suominen, a escola do futuro destina-se a abrir novos horizontes, ajudar os alunos a orientar-se no mundo das possibilidades, inspirar, motivar e aumentar a curiosidade e a paixão pelo desenvolvimento pessoal (VLADYKINA apud. SUOMINEM, 2015) onde o aprendizado possa ocorrer em muitos lugares, e as pessoas cada vez mais aprendendo uma com as outras, aprendendo de modo colaborativo (GROUP X, 2018).

Figura 1 - Blueprint da Lógica de Serviço em Arquitetura elaborado pelo Group X, Aalto University.

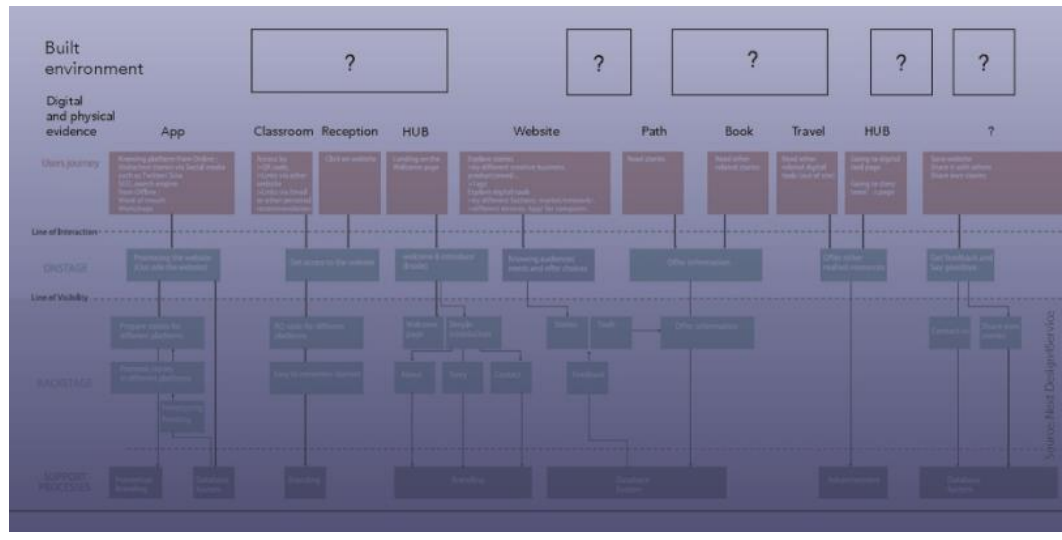

Fonte: GROUP X (2018)

Aquela prática contou com séries de entrevistas, workshops e propostas onde se buscou a elaboração de princípios de espaço para a solução conceitual de uma escola temporária permanente. Segundo Antti Ahlava apesar de as instalações serem temporárias e até certo ponto inadequadas, as crianças surpreendentemente gostavam delas e sentiam-se como se estivessem em casa. Eram ambientes considerados agradáveis pelas crianças onde possuíam contato visual com a área externa de maneira mais próxima entre outras características que tornavam as relações mais sociáveis entre os usuários (GROUP X, 2018). Segundo Vladykina esta experiência contribui com a teoria de que a eficiência no aprendizado é possível em espaços informais (VLADYKINA, 2016).

Durante o curso, os alunos serão familiarizados com o conceito de arquitetura de serviços, desenvolverão um método de utilização de um plano de serviços em programação arquitetônica durante o projeto de serviços e serão solicitados a projetar novas tipologias e soluções arquitetônicas (GROUP X, 2018).

A proposta desenvolvida durante o estudo traz abordagens de um design preocupado com interface na qual o usuário se relaciona com a edificação onde ferramentas como o blueprint service, - focado na prestação do serviço - são aplicadas com a finalidade de gerar a programação arquitetônica mapeando os hábitos do usuário e desse modo determinando com maior precisão o 
comportamento de cada perfil de indivíduo (figura 1). A aplicação desta ferramenta em arquitetura poderia permitir que ambientes projetados tenham seus programas definidos de modo mais otimizado, com melhor aproveitamento de espaços, melhorando a experiência do usuário e sua relação com a ambiente construído, combinando atividades e funções a partir de um melhor desenho da interface usuário-edifício. Desse modo seria possível talvez compreendermos a edificação como um ambiente verdadeiramente mais híbrido e amistoso ao usuário.

\section{Design de Serviço no Design de Interiores.}

O estudo em desenvolvimento está em aplicação no componente curricular intitulado 'Interiores IV - Serviços e Mobiliário' alocada no final do Curso Superior de Tecnologia em Design de Interiores do Centro Universitário de João Pessoa. Neste componente os alunos têm como objetivo "desenvolver as possibilidades de aplicação do design enquanto meio para a elaboração de ambientes de domínio público híbridas e alinhadas a novas influências tecnológicas na requalificação dos ambientes internos" (UNIPÊ, 2016). O desafio proposto aos alunos foi o de desenvolvimento de uma proposta de requalificação a partir de análise e síntese fundamentada na Lógica Dominante de Serviço para um ambiente institucional de ensino de domínio público. As etapas definidas para este componente estão divididas em vinte semanas organizadas da conforme quadro 1.

Quadro 1 - Cronograma de etapas/atividade do componente curricular.

\begin{tabular}{|c|c|c|c|c|c|c|c|c|c|c|c|c|c|c|c|c|c|c|c|c|}
\hline ETAPAS/SEMANAS & 01 & 02 & 03 & 04 & 05 & 06 & 07 & 08 & 09 & 10 & 11 & 12 & 13 & 14 & 15 & 16 & 17 & 18 & 19 & 20 \\
\hline \multicolumn{21}{|l|}{ IMERSÃO } \\
\hline \multicolumn{21}{|l|}{ Apresentação proposta } \\
\hline \multicolumn{21}{|l|}{ Seminário } \\
\hline \multicolumn{21}{|l|}{ Usuários/Visita Técnica } \\
\hline \multicolumn{21}{|l|}{ Workshop stakeholders } \\
\hline \multicolumn{21}{|l|}{ IDEAÇÃO } \\
\hline \multicolumn{21}{|l|}{ Análise/síntese workshop } \\
\hline \multicolumn{21}{|l|}{ Geração de proposta } \\
\hline \multicolumn{21}{|l|}{ Workshop propostas } \\
\hline \multicolumn{21}{|l|}{ Síntese propostas } \\
\hline \multicolumn{21}{|l|}{ PROTOTIPAÇÃO } \\
\hline \multicolumn{21}{|l|}{ Construção } \\
\hline \multicolumn{21}{|l|}{ Interação } \\
\hline \multicolumn{21}{|l|}{ Análise/síntese workshop } \\
\hline \multicolumn{21}{|l|}{ ANTEPROJETO } \\
\hline \multicolumn{21}{|l|}{ Desenvolvimento } \\
\hline Conclusão/Proposta Final & & & & & & & & & & & & & & & & & & & & \\
\hline
\end{tabular}

Fonte: autores (2018)

As etapas do componente foram planejadas seguindo as fases do design thinking para o desenvolvimento do projeto: Imersão, análise e síntese, ideação e prototipação. Em cada fase foram trabalhados conhecimentos, habilidades e atitudes específicas, a fim de capacitar o aluno a alcançar os objetivos esperados. Os conhecimentos esperados para a etapa de imersão é compreensão da relação entre usuário e espaço na construção de novas dinâmicas sociais aplicadas ao espaço interior como ambiente de serviço, através das teorias do design de serviço. Para este fim, foram ministradas aulas expositivas e dialogadas com a temática de design de serviços, quando foram 
abordadas definições básicas e seus princípios fundamentais, além de estudos de casos de aplicação em design de interiores.

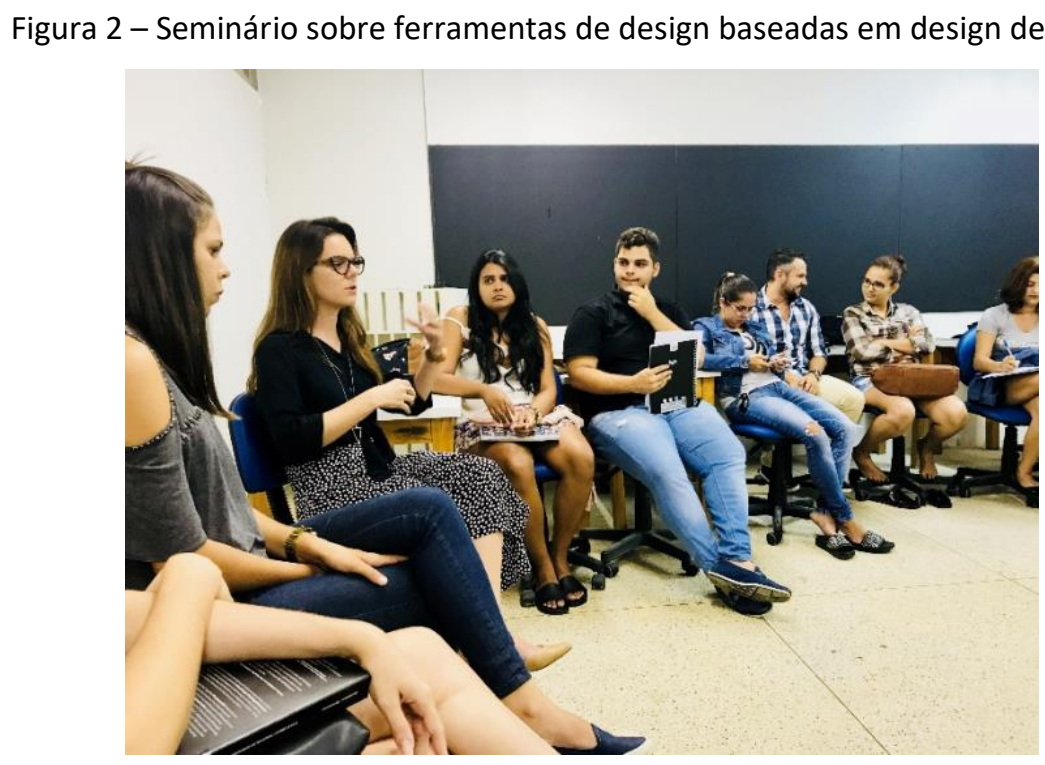

Fonte: autores (2018)

A fim de desenvolver no aluno a habilidade de identificar através de métodos de pesquisa as necessidades do projeto correlacionando às tendências, técnicas e tecnologias, adequadas ao usuário, foi utilizada a metodologia de sala de aula invertida para alinhamento conceitual e prático (figura 2). Na oportunidade, foi realizado debate dirigido pelos professores em que os alunos eram estimulados a explanar sobre as diferentes ferramentas de design de serviços e maneiras de aplicalas em projetos de interiores. Foram discutidas as ferramentas de mapa de stakeholders, mapa de jornada do usuário, os 5 porquês, um dia na vida, personas, criação de cenários, encenação de serviços e cocriação a fim de subsidiar o aluno no processo de imersão, análise e síntese e ideação. Esta atividade possibilitou que os docentes desenhassem objetivos tangíveis para o grupo, e ainda a partir de uma condução similar a um focus group possibilitou também que fossem identificados perfis de discentes adequados aos papeis que seriam necessários para a continuidade da atividade.

Para que a prática proposta no componente tivesse o máximo de isenção, o ambiente em estudo apenas foi apresentado aos discentes no momento do levantamento de campo. Foi solicitado que em determinado dia específico para este fim, todos os integrantes do grupo viessem à instituição preparados para o levantamento de campo. Ao chegar todos foram encaminhados ao Espaço de Vivência Acadêmica - EVA (figura 3 e 4). O EVA é considerado por muitos o prédio central do campus, ainda que não localizado em seu epicentro. Este fator se deve ao EVA abrigar a secretaria geral de ensino, restaurantes universitários, e alguns departamentos institucionais, bem como lojas e serviços dedicados aos alunos, professores e colaboradores. Contudo, é ponderado que muitas das atividades desempenhadas nesta edificação não atribuem a arquitetura a condição de um ambiente que fortaleza as relações de convívio. Observadas estas condições elegeu-se por esta edificação devido ao seu bom potencial de investigação e geração de propostas. 
Figura 3 - Vista do Espaço de Vivência Acadêmica.

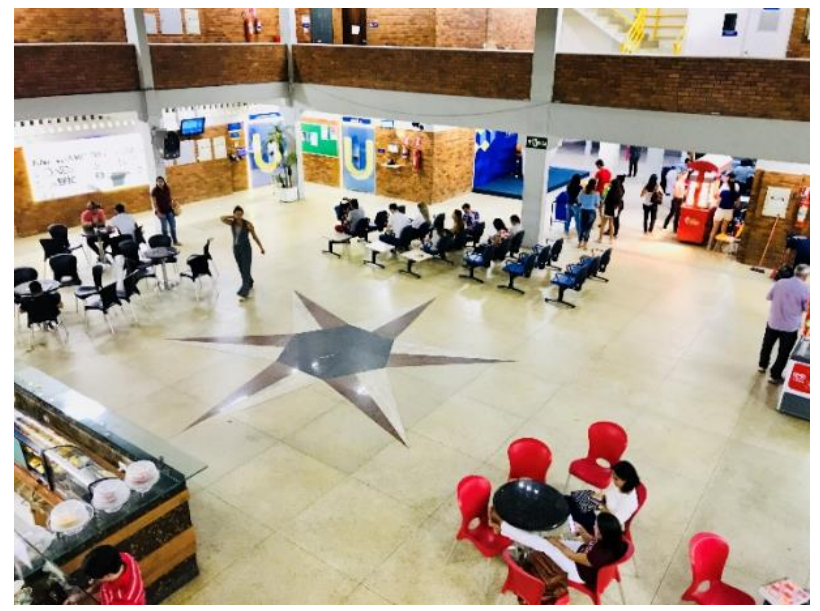

Fonte: autores (2018).
Figura 4 - Espaço de Vivência Acadêmica

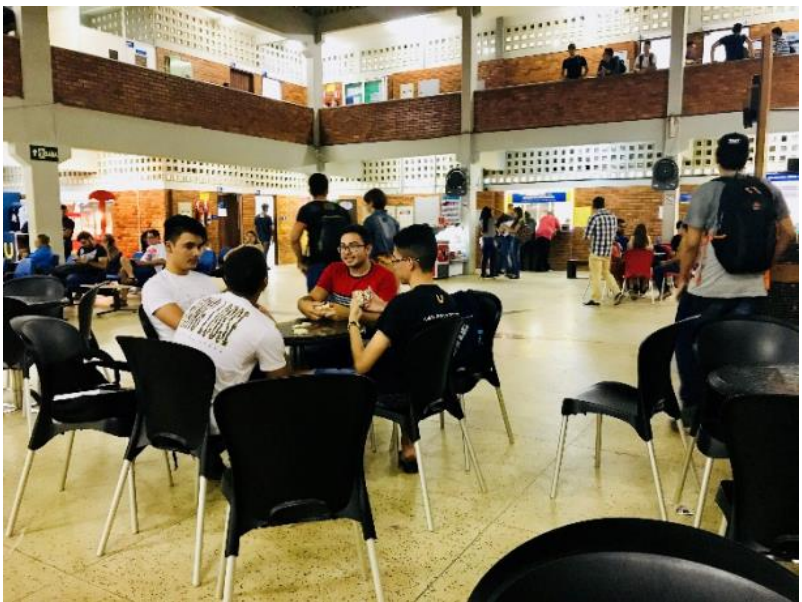

Fonte: autores (2018).

Ainda na etapa de imersão, a visita técnica objetivou desenvolver no aluno a atitude de manter o habito da observação ativa e participante na busca do aprimoramento técnico. Foram feitas medições, observações, breves entrevistas com usuários e registros fotográficos. A fim de desenvolver a capacidade de mobilizar conhecimentos adquiridos em componentes anteriores aplicáveis nas práticas de projeto, e empregar diferentes métodos de análise na definição das diretrizes de projeto, foi dirigido um novo debate em sala de aula para o compartilhamento e alinhamento do material coletado e das experiências vivenciadas.

Figura 5 - Workshop com os stakeholders.

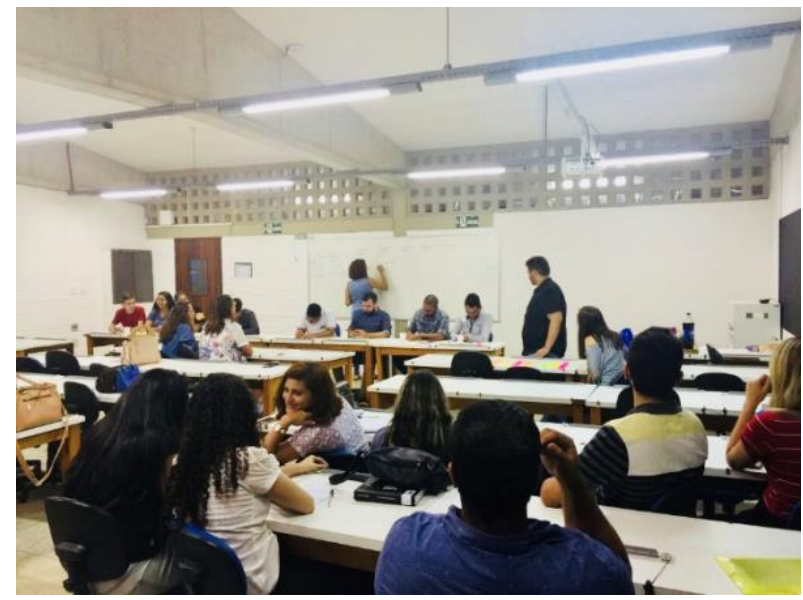

Fonte: autores (2018).
Figura 6 - Construção do Mapa de Jornada do Usuário

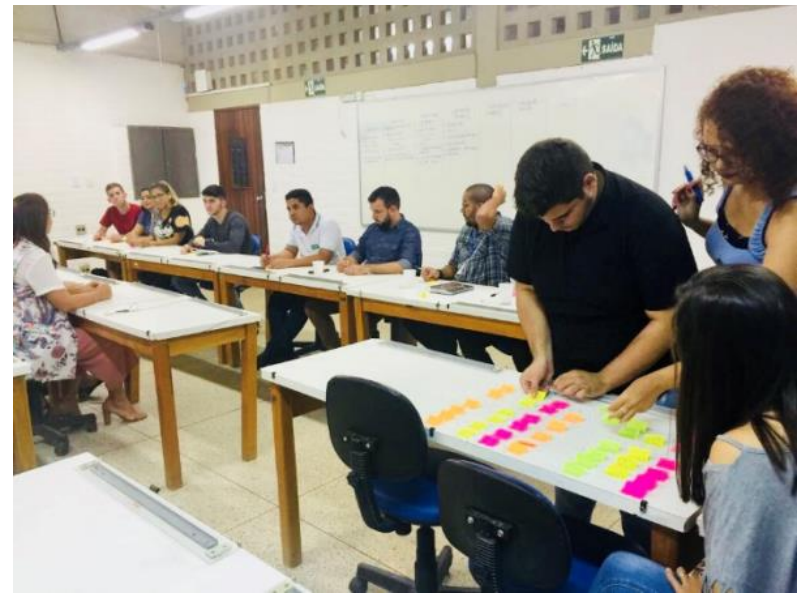

Fonte: autores (2018).

Em sequência, foi promovido um workshop com os stakeholders para o aprofundamento no processo de imersão (figura 5). Foram convidados atores envolvidos nas atividades que ocorrem no espaço de intervenção: alunos de diferentes cursos, colaboradores da instituição, professores e coordenadores. Com a supervisão dos professores, os alunos promoveram debates com os stakeholders, administraram entrevistas, aplicaram as ferramentas: 5 porquês, mapa de stakeholders, mapa de jornada do usuário, entre outras (figura 6). Foram feitos registros fotográficos e de áudio. Na ocasião foi possível conhecer o perfil dos atores, as motivações práticas 
e emocionais para utilização do ambiente em estudo, suas frustações e anseios frutos das experiências que já vivenciadas no espaço, e desejos na manutenção de uma relação mais agradável com o entorno.

Ao fim do workshop com stakeholders, foi iniciada a fase de análise e síntese, quando foi analisado o material coletado na imersão a fim de serem definidas as diretrizes do projeto. A análise do material foi trabalhada coletivamente, com intuito de estimular a cocriação. Para síntese dos dados foi desenvolvido um blueprint com a descrição e definição dos diferentes elementos e processos presentes nos serviços realizados no espaço. As atividades do workshop e blueprint favoreceram a troca de conhecimentos e experiências entre os stakeholders e alunos, além de promover a produção colaborativa de insights criativos. A partir da síntese do material coletado, os alunos foram orientados a definirem individualmente diretrizes projetuais, visto que a continuidade do estudo se dará de modo individual.

A fase de ideação objetivou desenvolver no aluno o conhecimento de definição de soluções técnicas e tecnológicas inovadoras na concepção de ambientes compartilhados visando a otimização do uso. Para tal, foi trabalhada a setorização e zoneamento dos espaços, desenvolvimento de layout com identificação de ambientes, dimensões, atributos e funções dos espaços. Estas práticas visaram estimular no discente a habilidade de planejar ambientes a partir dos preceitos do design de serviço.

Com o cumprimento das fases de imersão, análise e síntese, e o início da fase de ideação, foram realizadas na primeira verificação de aprendizagem, na qual os alunos foram orientados a apresentarem tecnicamente seus projetos. As atividades em desenvolvimento se encontram ainda na fase de ideação. Nas etapas subsequentes serão trabalhadas a continuidade da ideação, prototipação e teste, além da especificação detalhada do projeto, a fim de aperfeiçoar no discente as habilidades de domínio técnico de representação gráfica em projeto executivo e detalhamento construtivo. Pretende-se realizar um novo workshop com stakeholders, desta vez para teste das soluções gerados pelos alunos.

\section{Pretensões além da conclusão da atividade}

Pretende-se a partir do aprofundamento deste estudo construir um entendimento filosófico sobre os métodos de desenvolvimento de projeto adotados no caso estudado. Inicialmente, o domínio metodológico desta abordagem poderá corroborar com o aperfeiçoamento das práticas desenvolvidas no Curso Superior de Tecnologia em Design de Interiores do Centro Universitário de João Pessoa - Unipê.

De modo mais abrangente espera-se que o aperfeiçoamento de técnicas e ferramentas de análise e síntese como algumas estudadas nesta componente curricular possibilitem maior eficiência na definição de atributos para programas arquitetônicos permitindo reflexões acerca de tipologias arquitetônicas.

\section{Considerações finais}

Consideramos inicialmente que a propositura de disciplinas práticas investigativas permanentes na carreira acadêmica, que busquem a experimentação, análise ou comprovação de fatos através de referências científicas comprovadas no mundo real como estudos de caso e ou mesmo outros experimentos, podem contribuir fortemente na condição de formação do profissional egresso permitindo que conhecimentos de vanguarda chegam de maneira mais rápida 
e eficiente ao mercado. Como ponto complementar consideramos importantíssimo apontar o relevante entusiasmo dos discentes como reflexo de práticas experimentais em ambiente acadêmico onde o docente se coloca em posição de pesquisador junto ao discente.

Acerca das práticas metodológicas e da abordagem em lógica de serviço consideramos que a continuidade da investigação se faz necessária para que os resultados possam ser mensurados em sua integridade. Contudo, já se pode considerar que a abordagem em serviço aplicada ao ambiente construído pode contribuir significativamente na proposição de novas estruturas ou mesmo na requalificação de ambientes. Desse modo, acreditamos que além do limite da aprendizagem seja oportuno iniciar um grupo de estudo ou mesmo um projeto de pesquisa a fim de melhorar o entendimento quanto a aplicação dos métodos de análise propostos. De todo modo, já vem se observando que a aplicação desta abordagem em arquitetura pode permitir que ambientes projetados tenham seus programas definidos de modo mais otimizado, com melhor aproveitamento de espaços, sobrepondo funções e atividades a partir de um melhor desenho da interface usuário-edificação. Dessa forma seria possível talvez compreendermos a edificação como um ambiente verdadeiramente mais híbrido.

\section{Referências}

GROUP X. Service Logic in Architecture. In: Group X Aalto University, 2018. (http://www.groupxaalto.fi/portfolio/service-logic-in-architecture/).

Labs for Learners Studio Course. In: Group X Aalto University, 2018. (http://www.groupxaalto.fi/portfolio/labs-for-learners-studio-course/).

Co-School Experimentation Labs Rethinking Learning. In: Group X Aalto University, 2018. (http://www.groupxaalto.fi/2016/08/school-as-a-service-interview/).

STICKDORN, Marc. Isto é Design Thinking de Serviços. Porto Alegre: Bookman 2014.

SUOMINEN, Jarmo. School as a Service, Concept Idea. 2015

UNIPÊ. Projeto Pedagógico de Curso Superior de Tecnologia em Design de Interiores. Centro Universitário de João Pessoa, 2016.

VIANNA, Maurício et al. Design Thinking: inovação em negócios. Rio de Janeiro: MJV Press, 2012.

LUSCH, Robert F; VARGO, Stephen L.; GUSTAFSSON, Anders. Fortering a trans-disciplinary perpectives of service ecosystems. Journal of Business Research 69 - 2016 (p.2957-2963)

VLADYKINA, Natalia. Service-Dominant Logic in the Spatial Design of a School - Master's Thesis. Aalto University. School of Art, Design and Architecture. Department of Design - 2016. 